\title{
SETDB1 induces epithelial-mesenchymal transition in breast carcinoma by directly binding with Snail promoter
}

\author{
WENLIN YANG ${ }^{1}$, YING SU ${ }^{2}$, CHENJIAN HOU ${ }^{2}$, LINLIN CHEN $^{3}$, DANMEI ZHOU ${ }^{2}$, \\ KEHAN REN ${ }^{2}$, ZHAOPING ZHOU ${ }^{4}$, RENYA ZHANG ${ }^{1}$ and XIUPING LIU ${ }^{3}$ \\ ${ }^{1}$ Department of Pathology, Affiliated Hospital of Jining Medical University, Jining Medical University, Jining, \\ Shandong 272029; ${ }^{2}$ Department of Pathology, School of Basic Medical Sciences, Fudan University, Shanghai 200032; \\ ${ }^{3}$ Department of Pathology, The Fifth Hospital of Shanghai, Fudan University, Shanghai 200240; \\ ${ }^{4}$ Department of General Surgery, Huashan Hospital, Fudan University School of Medicine,
} Shanghai 200040, P.R. China

Received June 27, 2018; Accepted November 8, 2018

DOI: 10.3892/or.2018.6871

\begin{abstract}
SET domain bifurcated 1 (SETDB1) is a histone $\mathrm{H} 3$ lysine 9 methyltransferase that is highly expressed in various tumor types, including breast cancer. However, how SETDB1 functions in breast cancer is unclear. In the present study, proliferation, migration and invasion assays were performed to explore the role of SETDB1 in breast cancer cells. SETDB1 downregulation in BT549 and MDA-MB-231 cells reduced cell proliferation, whereas upregulation in MCF7 and T47D cells enhanced proliferation. Depletion of SETDB1 suppressed cell migration and invasion in vitro and reduced lung metastasis in vivo. By contrast, SETDB1 overexpression enhanced cell migration and invasiveness. Notably, SETDB1 overexpression appeared to induce epithelial-mesenchymal transition (EMT) in MCF7 cells. Mechanistic investigations indicated that SETDB1 acts as an EMT inducer by binding directly to the promoter of the transcription factor Snail. Thus, SETDB1 is involved in breast cancer metastasis and may be a therapeutic target for treating patients with breast cancer.
\end{abstract}

\section{Introduction}

Breast cancer is the most common cancer among women and a global public health crisis (1). This cancer readily metastasizes, which limits further treatment (2). Therefore,

Correspondence to: Professor Renya Zhang, Department of Pathology, Affiliated Hospital of Jining Medical University, Jining Medical University, 89 Guhuai Road, Jining, Shandong 272029, P.R. China

E-mail: hzzhang_1964@163.com

Professor Xiuping Liu, Department of Pathology, The Fifth Hospital of Shanghai, Fudan University, 128 Ruili Road, Minhang, Shanghai 200240, P.R. China

E-mail: xpliu1228@fudan.edu.cn

Key words: SET domain bifurcated 1, Snail, epithelial-mesenchymal transition, breast cancer the molecular mechanisms underlying breast cancer must be better understood. Epigenetic alterations are fundamental for the formation and progression of cancer, and histone modifications, such as histone methylation, are associated with breast cancer prognosis (3). Histone methylation commonly occurs at histone $3(\mathrm{H} 3)$ and $\mathrm{H} 4$ arginine or lysine residues, and is catalyzed by an enzyme family with a conserved catalytic domain or su(var)3-9, enhancer-of-zeste and trithorax (SET) domain (4).

The SET domain bifurcated 1 (SETDB1) gene is located on human chromosome 1q21 and encodes a histone H3 lysine 9 methyltransferase, which is also known as ESET or KMT1E (5). SETDB1 can be targeted to the KRAB-associated protein-1 (KAP-1) corepressor and enhance binding of heterochromatin protein 1 proteins that contribute to gene expression silencing $(6,7)$. SETDB1 is also involved in transcriptional repression and heterochromatin formation with methyl-CpG-binding domain protein 1 and the human homologue of mouse ATFa-associated modulator (8-10). Studies suggest that SETDB1 is essential for early embryonic development and maintenance of embryonic stem cells (11-15). SETDB1 was previously identified as an oncogene in melanoma (16). Subsequently, SETDB1 was demonstrated to be overexpressed and required for cancer cell proliferation and metastasis in various solid tumors, including glioma, and prostate, lung and colorectal cancer (17-21). Furthermore, the promoter activity of SETDB1 was inhibited by p53 protein, leading to paclitaxel induced-cell death in human lung cancer cells (22). These findings suggest that SETDB1 has an important role in cancer cell growth and metastasis, and in drug resistance. Thus, as a H3K9 special histone methyltransferase, SETDB1 may be a promising epigenetic target for clinical treatment $(23,24)$.

In the present study, SETDB1 overexpression was demonstrated to increase breast cancer cell proliferation, migration and invasion, and depletion of SETDB1 decreased metastasis in vivo. Furthermore, SETDB1 upregulation induced epithelial-mesenchymal transition (EMT) in MCF7 cells. Finally, SETDB1 was identified as an EMT inducer that binds directly to the promoter of the Snail transcription factor. 


\section{Materials and methods}

Cell culture. The cell lines MCF7, T47D, BT549 and MDA-MB-231 breast cancer were obtained from the Type Culture Collection of Chinese Academy of Sciences (Shanghai, China). MCF7 and M231 cells were maintained in Dulbecco's modified Eagle's medium (Gibco; Thermo Fisher Scientific, Inc., Waltham, MA, USA). T47D and BT549 cells were maintained in RPMI-1640 medium (Gibco; Thermo Fisher Scientific, Inc.). All media were supplemented with $10 \%$ fetal bovine serum (FBS; Biowest, SAS, Nuaille, France) and cell lines were cultured at $37^{\circ} \mathrm{C}$ with $5 \% \mathrm{CO}_{2}$.

Western blot analysis. Total protein was extracted from cells using a radioimmunoprecipitation lysis buffer (cat. no. sc-24948; Santa Cruz Biotechnology, Inc., Dallas, TX, USA). A bicinchoninic acid protein Assay (Thermo Fisher Scientific, Inc.) was used to quantify total protein concentration. Equal amounts of proteins $(50 \mu \mathrm{g} /$ well) were separated by SDS-PAGE on $10 \%$ gels and electrophoretically transferred onto polyvinylidene fluoride membranes (cat. no. IPFL00010; Merck KGaA, Darmstadt, Germany). The membranes were blocked with 5\% milk in Tris- $\mathrm{HCl}$ buffered solution for $2 \mathrm{~h}$ at room temperature, and then incubated overnight at $4{ }^{\circ} \mathrm{C}$ with primary antibodies and secondary antibody (1:2,000, cat. no. 7074; Cell Signaling Technology, Inc., Danvers, MA, USA) at $37^{\circ} \mathrm{C}$ for $1 \mathrm{~h}$. The signals were visualized using an enhanced chemiluminescent substrate (Thermo Fisher Scientific, Inc.) and detected by a FluorChem Q imaging system (ProteinSimple, San Jose, CA, USA). Images from western blot analysis were quantified using Quantity One ${ }^{\circledR}$ software 4.6.6 (Bio-Rad Laboratories, Inc., Hercules, CA, USA). The expression level was normalized with respect to an internal control. Primary antibody against SETDB1 was obtained from ProteinTech Group, Inc. (Chicago, IL, USA; cat. no. 11231-1-AP), and primary antibodies against E-cadherin (cat. no. 3195), $\beta$-catenin (cat. no.8480), vimentin (cat. no. 5741), Snail (cat. no. 3879) and Slug (cat. no. 9585) were obtained from Cell Signaling Technology, Inc. (EMT Antibody Sampler Kit; cat. no. 9782). $\beta$-actin rabbit monoclonal antibody $(\mathrm{mAb})$ internal control was obtained from Cell Signaling Technology, Inc. (cat. no. 8457).

Reverse transcription-quantitative polymerase chain reaction $(R T-q P C R)$. Total RNA was obtained using RNAiso plus (cat. no. 9108; Takara Biotechnology Co., Ltd., Dalian, China), and $2 \mu \mathrm{g}$ total RNA was reversed transcribed to cDNA using PrimeScript RT Master Mix (cat. no. DRRO36A; Takara Biotechnology Co., Ltd.) according to the manufacturer's instructions. Primers used to detect SETDB1 (forward, 5'-GATGAGGAACTGGAGAAGATG-3' and reverse, 5'-ATT AGTCACTGCCCTGGATG-3'); transforming growth factor 1 (TGF1 forward, 5'-ACCTGAACCCGTGTTGCTCT-3' and reverse, 5'-GAACCCGTTGATGTCCACTT-3'); zinc finger E-box binding homeobox 1 (ZEB1 forward, 5'-GAAAAT CCAGTCGCTACAA-3' and reverse, 5'-CACACAGAAGAC AAGTGCTA-3'); Snail (forward, 5'-CCCAATCGGAAG CCTAACT-3' and reverse, 5'-GCTGCTGGAAGGTAAACT CT-3'); Slug (forward, 5'-CTCAGAAAGCCCCATTAGT-3'; reverse, 5'-GCCCAGAAAAAGTTGAATAG-3'); tight junction protein 1 (ZO-1 forward, 5'-GCAGCAAGAGATGGC
AATA-3' and reverse, 5'-CAGGGACATTCAATAGCGTA); and GAPDH (internal control forward, 5'-CTGACTTCAACA GCGACACC-3' and reverse, 5'-TGCTGTAGCCAAATTCGT TGT-3'); transcripts were obtained from BioTNT (Shanghai, China). qPCR was performed using SYBR Premix Ex Taq (cat. no. RR420A; Takara Biotechnology Co., Ltd.) on a StepOnePlus Real-Time System (Applied Biosystems; Thermo Fisher Scientific, Inc.). The thermal cycle conditions consisted of one cycle at $95^{\circ} \mathrm{C}$ for $10 \mathrm{~min}$, followed by 40 cycles of amplification at $95^{\circ} \mathrm{C}$ for $15 \mathrm{sec}$, and then $60^{\circ} \mathrm{C}$ for $1 \mathrm{~min}$. The expression level of mRNA was obtained by calculating the $2^{-\Delta \Delta \mathrm{Cq}}$ values (25)

Lentiviral-vector infections. pLVX-SETDB1-wt-IRES-Puro and pLKD-EF1a-EGFP-P2A-LUC-F2A-Puro-U6 shSETDB1 vectors were generated by Obio Technology (Shanghai) Co., Ltd. (Shanghai, China). The empty vector acts as control group. The three shRNA sequences were as follows: S1, 5'-GGGCAGTGACTAATTGTGA-3'; S2, 5'-GCATGCGAA TTCTGGGCAAGA-3'; S3, 5'-GGGAGGACATAGAAGACA TCT-3'. The negative control shRNA (shNC) sequences were as follows: Y009 forward, 5'-CCGGTTCTCCGAACGTGTCAC GTTTCAAGAGAACGTGACACGTTCGGAGAATTTTTT G-3'; Y009 reverse, 5'-AATTCAAAAAATTCTCCGAAC GTGTCACGTTCTCTTGAAACGTGACACGTTCGGAGA A-3'. Cells $\left(5 \times 10^{4}\right)$ were seeded onto 24 -well plates. Lentiviral vectors were introduced into breast cancer cells at different multiplicity of infection (MOI) values (MDA-MB-231 and BT549, MOI 10; MCF7 and T47D, MOI 40). Different final concentrations $(2,4,6,8$ and $10 \mu \mathrm{g} / \mathrm{ml})$ of puromycin were applied to the breast cancer cells for $24 \mathrm{~h}$, and the concentration of $6 \mu \mathrm{g} / \mathrm{ml}$ was selected to kill non-transfected cells for 2 weeks to obtain stably expressed cells. Transfection efficiency was validated with RT-PCR and western blot analysis.

Cell proliferation assays. A total of $2 \times 10^{3}$ cells/well were cultured in 96-multiwell plates for $96 \mathrm{~h}$ or 7 days. Cells were incubated with CCK-8 reagent (10 $\mu \mathrm{l} /$ well; cat. no. CK04-11; Dojindo Molecular Technologies, Inc., Kumamoto, Japan) for $1 \mathrm{~h}$ at $37^{\circ} \mathrm{C}$. Absorbance at $450 \mathrm{~nm}$ was read using a Multiscan Go-1510 microplate reader (Thermo Fisher Scientific, Inc.).

Migration and invasion assay. Cells $\left(\sim 5 \times 10^{4}\right)$ were resuspended in $100 \mu \mathrm{l}$ serum-free medium and plated in the upper chamber of a Transwell assay system (cat. no. 3422; Corning Incorporated, Corning, NY, USA). Then, 10\% FBS-containing medium was added to the lower chamber. For the invasion assay, the Transwell membrane was precoated with Matrigel (cat. no. 354234; BD Biosciences, Franklin Lakes, NJ, USA) and incubated at $37^{\circ} \mathrm{C}$ for $4 \mathrm{~h}$. After cells were cultured for $24 \mathrm{~h}$, the upper chambers were fixed with $100 \%$ methanol for $10 \mathrm{~min}$ at room temperature, and stained with $0.1 \%$ crystal violet for $20 \mathrm{~min}$ at room temperature. Cells were counted under a light microscope in five predetermined fields and averaged.

Wound healing assay. Cells $\left(\sim 5 \times 10^{5}\right)$ were seeded onto 6-well plates and incubated overnight. A 1,000 $\mu$ l sterile pipette tip was used to scratch the cell monolayer (point of zero migration). After cell incubation for 24 and $48 \mathrm{~h}$, phase-contrast images of 
the scratched area were captured using computer-assisted light microscopy to assess gap closure.

Animal experiments. Female nude mice aged 4-6 weeks $(n=12)$ were obtained from Shanghai SLAC Laboratory Animal Co. Ltd. (Shanghai, China). Cells $\left(\sim 1 \times 10^{5}\right.$ cells in $\left.100 \mu \mathrm{l}\right)$ were resuspended in serum-medium and injected into the tail vein of mice. The animals were monitored twice weekly. Body condition score was used to assessment of overall health of the animal from 1 (emaciated/wasted) to 5 (obese) (25). After 2 months of observation, the maximum body weight loss was $5 \%$ of original weight. When the body condition score was $1 / 5$, all mice were euthanized and organs were dissected. Lungs were fixed with $10 \%$ buffered formalin for $24 \mathrm{~h}$ at room temperature, and embedded in paraffin. Sections ( $4 \mu \mathrm{m}$-thick) were dewaxed in xylene, rehydrated through decreasing concentrations of ethanol (100, 95 and 80\%) and washed in PBS. Then stained with hematoxylin for $10 \mathrm{~min}$ and eosin for $30 \mathrm{sec}$ at room temperature. Following staining, sections were dehydrated through increasing concentrations of ethanol $(80$, 95 and 100\%) and xylene. Animal experiments were approved by the Ethics Committee of Fudan University (Shanghai, China).

Chromatin immunoprecipitation (CHIP) assay. CHIP assay was performed with a SimpleCHIP Enzymatic Chromatin IP kit (cat. no. 9003; Cell Signaling Technology, Inc.) according the manufacturer's instructions. Briefly, $\sim 4 \times 10^{6} \mathrm{MCF} 7$ cells overexpressing SETDB1 for each immunoprecipitation (IP) were crosslinked with $1 \%$ formaldehyde at room temperature for $10 \mathrm{~min}$. Then, $0.5 \mu \mathrm{l}$ micrococcal nuclease was added/IP preparation to digest DNA to $150-900 \mathrm{bp}$. IP antibodies histone H3 (D2B12) XP Rabbit mAb (10 $\mu$; positive control; cat. no. 4620), normal rabbit $\operatorname{IgG}(2 \mu \mathrm{l}$; negative control; cat. no. 2729) and SETDB1 rabbit polyclonal antibody (2 $\mu \mathrm{g}$; cat. no. 11231-1-AP; Proteintech Group, Inc.) were added to $500 \mu \mathrm{l} 1 \mathrm{X}$ CHIP buffer containing $10 \mu \mathrm{g}$ fragmented chromatin DNA at $4^{\circ} \mathrm{C}$ with rotation overnight. Then, $30 \mu \mathrm{l}$ CHIP-Grade Protein G Magnetic Beads (cat. no. 9006; Cell Signaling Technology, Inc.) were used to pull-down the antibody/chromatin complex at $4^{\circ} \mathrm{C}$ with rotation for $2 \mathrm{~h}$. Chromatin was eluted from the antibody/protein $\mathrm{G}$ magnetic beads at $65^{\circ} \mathrm{C}$ for $30 \mathrm{~min}$ and reversed cross-linked with $6 \mu \mathrm{l}$ $5 \mathrm{M} \mathrm{NaCl}$ and $2 \mu \mathrm{l}$ proteinase $\mathrm{K}$ (cat. no. 10012) at $65^{\circ} \mathrm{C}$ for $2 \mathrm{~h}$. DNA was purified using spin columns (centrifuged at $18,500 \mathrm{x} \mathrm{g}$ in a microcentrifuge for $30 \mathrm{sec}$.) and quantified by PCR. A master reaction mix was prepared including $12.5 \mu \mathrm{l}$ nuclease-free $\mathrm{H}_{2} \mathrm{O}, 2.0 \mu \mathrm{l} 10 \mathrm{X}$ PCR buffer, $1.0 \mu \mathrm{l} 4 \mathrm{mM}$ dNTP Mix, $2.0 \mu \mathrm{l} 5 \mu \mathrm{M}$ primers, $0.5 \mu \mathrm{l}$ Taq DNA Polymerase (cat.no. EP0404; Thermo Fisher Scientific, Inc.). The following PCR reaction program was used: Initial denaturation, $95^{\circ} \mathrm{C}$ $5 \mathrm{~min}$; denaturation, $95^{\circ} \mathrm{C} 30 \mathrm{sec}$; annealing, $62^{\circ} \mathrm{C} 30 \mathrm{sec}$; extension, $72^{\circ} \mathrm{C} 30 \mathrm{sec}$; denaturation, annealing and extension were repeated for a total of 34 cycles; final extension of $72^{\circ} \mathrm{C}$ 5 min. PCR products $(10 \mu \mathrm{l})$ were resolved on $2 \%$ agarose gels and ethidium bromide was used to visualize DNA. Six pairs of Snail primers were used (P2 forward, 5'-GGAAGCCAGCGT GAAAGATC-3' and reverse, 5'-TCCTCTCCTCAGCCAACT CG-3'; P3 forward, 5'-GAGAGGACTTTGGCTTTTAC-3' and reverse, 5'-TCATTAAGCGGAATACTCCC-3'; P4 forward,
5'-GTATTCCGCTTAATGACTGC-3' and reverse, 5'-AAA ACCTATAAGCACCCCAC-3'; P5 forward, 5'-GTGGTGTGG GGTGCTTATAG-3' and reverse, 5'-CTGTAACACGGC TCCATAGG-3'; P6 forward, 5'-AGCCGTGTTACAGCCTTT AG-3' and reverse, 5'-CGTAGGAGTTTGGACTTTGC-3'; P7 forward, 5'-AAAGTCCAAACTCCTACGAG-3' and reverse, 5'-ATTATCAAGGGAAAAGGCCC-3').

Knockout of Snail by clustered regularly interspaced short palindromic repeats (Crispr)/Cas9. Single guide RNA (sgRNA) was designed using an online Optimized Crispr Design Tool (genome-engineering.org). The Snail gene sequence was downloaded from Ensemble Genome Browser (ensembl.org). The 25 nucleotide sequences followed by protospacer adjacent motif sequence were designed as sgRNA (sense, 5'-CACCGTGTAGTTAGGCTTCCGATTG-3' and antisense, 5'-AAACCAATCGGAAGCCTAACTACAC-3'). The sequence of NC sgRNA was as follows: 5'-GTATTA CTGATATTGGTGGG-3'. Following phosphorylation and annealing $\left(37^{\circ} \mathrm{C}\right.$ for $30 \mathrm{~min}, 95^{\circ} \mathrm{C}$ for $5 \mathrm{~min}$, and then ramping down to $25^{\circ} \mathrm{C}$ at $5^{\circ} \mathrm{C} / \mathrm{min}$ ), the sgRNA oligos were cloned into a $B b s$ I-digested pX330 vector in a thermocycler $\left(37^{\circ} \mathrm{C}\right.$ for $5 \mathrm{~min}, 23^{\circ} \mathrm{C}$ for $5 \mathrm{~min}$ for a total of six cycles and then held at $4^{\circ} \mathrm{C}$ hold until analysis). Then, $1-2 \mu \mathrm{l}$ of the final product was transformed into DH5 $\alpha$ competent cells. Colonies were selected and verified by sequencing performed by Sangon Biotech Co., Ltd. (Shanghai, China). Cells $\left(5 \times 10^{4}\right)$ were seeded into 24 well plates. DNA $(1 \mu \mathrm{g})$ and $2 \mu$ l Lipofectamine ${ }^{\circledR} 3000$ (cat. no. L3000001; Thermo Fisher Scientific, Inc.) were added to cells for $24 \mathrm{~h}$.

Statistical analysis. Statistical analyses were performed using GraphPad Prism 6 software (GraphPad Software, Inc., La Jolla, CA, USA). Three independent experiments were performed. The data of western blot analyses were quantified using Quantity One ${ }^{\circledR}$ software 4.6.6 (Bio-Rad Laboratories, Inc., Hercules, CA, USA). Student's t-test was used to compare data the difference between two groups. One-way analysis of variance multiple comparison with Tukey's post hoc test was used for multiple groups. $\mathrm{P}<0.05$ was considered to indicate a statistically significant difference.

\section{Results}

SETDB1 promotes breast cancer cell growth in vitro. SETDB1 was overexpressed in the MCF7 and T47D cells, and knocked down in BT549 and MDA-MB-231 cells via lentiviral-vector infections. Transfection efficiency was validated by RT-qPCR and western blot analysis (Fig. 1A). Proliferation assays revealed that knocking down SETDB1 expression resulted in a decrease in the proliferation of BT549 cells at $84 \mathrm{~h}$ and at 24 $\mathrm{h}$ in MDA-MB-231 cells (Fig. 1B). By contrast, overexpression of SETDB1 increased proliferation of T47D cells after $84 \mathrm{~h}$ and MCF7 cells after 2 days (Fig. 1C).

SETDB1 downregulation inhibited breast cancer cell migration and invasion in vitro as well as metastasis in vivo. Transwell assay results revealed that downregulation of SETDB1 in BT549 (Fig. 2A) and MDA-MB-231 (Fig. 2B) cells significantly decreased migration and invasiveness, 
A
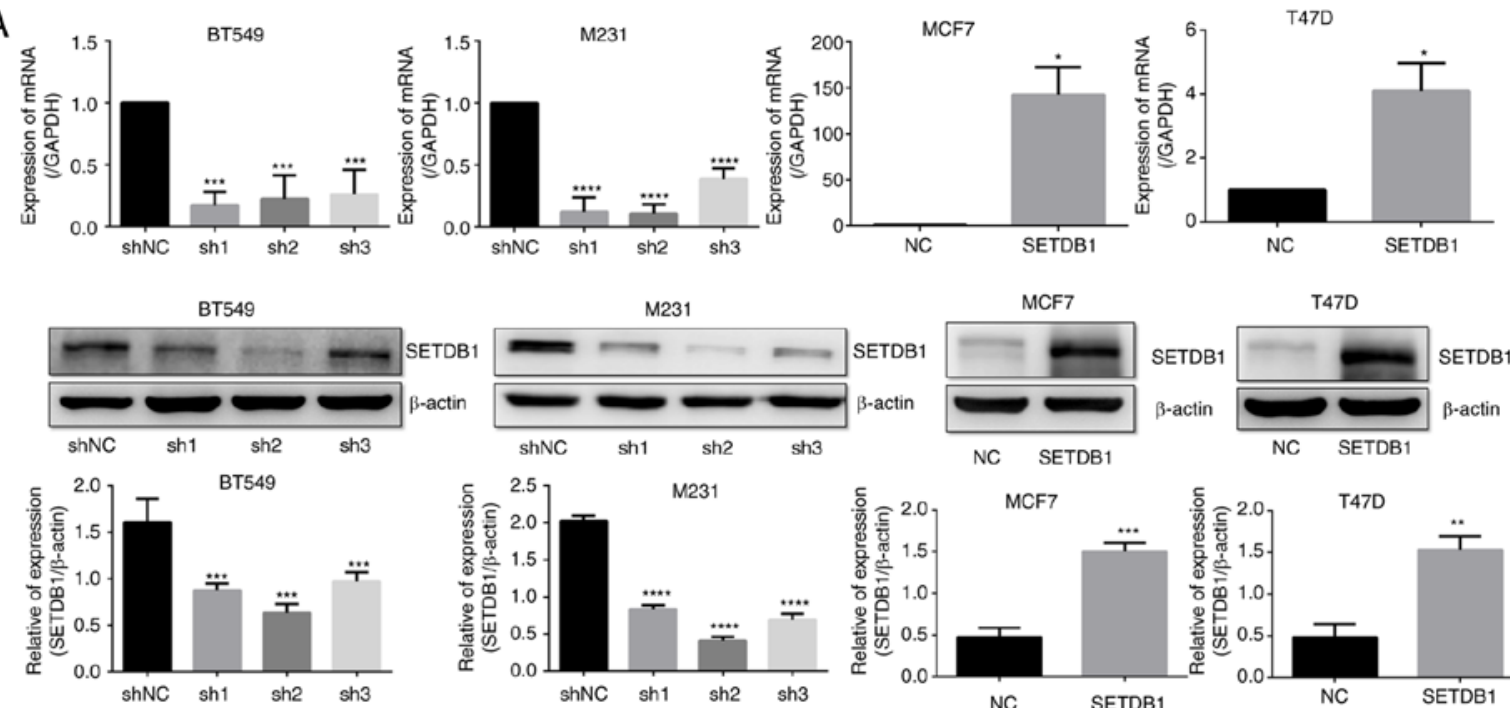

MCF7

B
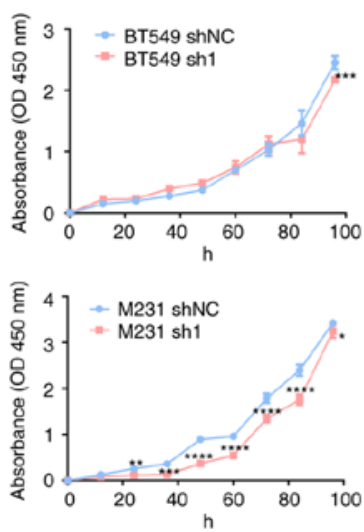
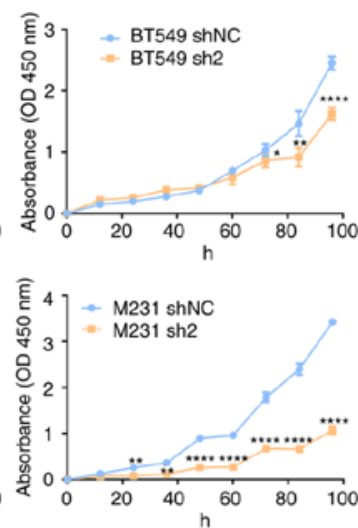
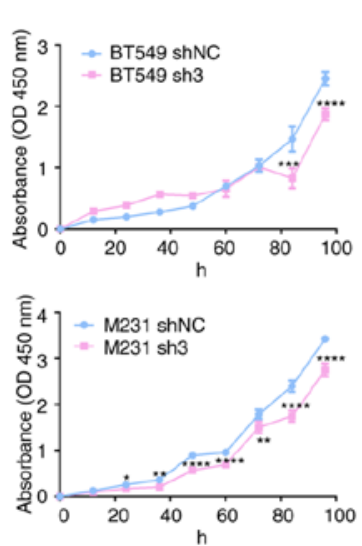

T47D
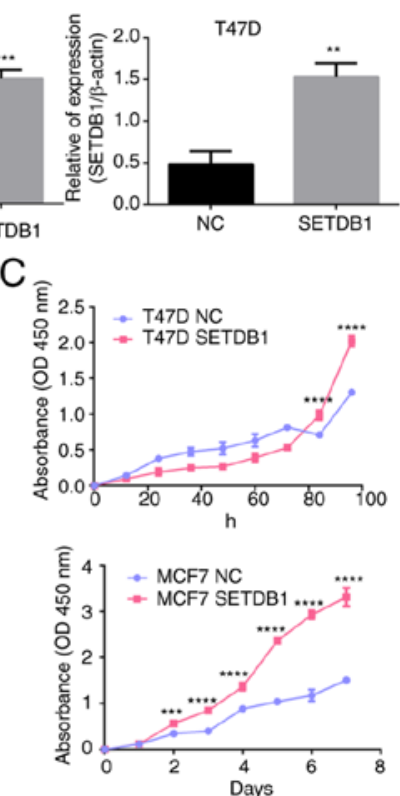

Figure 1. SETDB1 promotes breast cancer cell growth in vitro. (A) Lentiviral vector infections were performed to overexpress SETDB1 in the MCF7 and T47D cells and knock down SETDB1 in the BT549 and MDA-MB-231 cells. Transfection efficiency was validated by reverse transcription-quantitative polymerase chain reaction (upper) and western blot analysis (lower). (B) Downregulation of SETDB1 decreased proliferation in BT549 and MDA-MB-231 cells; mean \pm SD of three wells. (C) SETDB1 overexpression increased proliferation in T47D and MCF7 cells; mean \pm SD of three wells. ${ }^{*}<0.05$, ${ }^{* *} \mathrm{P}<0.01$, ${ }^{* * * *} \mathrm{P}<0.001,{ }^{* * * *} \mathrm{P}<0.0001$ vs. shNC or NC. SD, standard deviation; sh, short hairpin RNA; NC, negative control; SETDB1, SET domain bifurcated 1; OD, optical density.

particularly in breast cancer cells transfected with lentiviral containing the sh2 sequence compared with the negative control. Ectopic expression of SETDB1 facilitated migration and invasiveness in MCF7 cells (Fig. 2C). A wound-healing assay revealed that SETDB1 overexpression in MCF7 cells significantly increased cell migration compared with controls (Fig. 2D). In MDA-MB-231-treated immunodeficient mice, $H \& E$ staining revealed that multiple tumor nodules were observed in lungs of MDA-MB-231/NC animals (4/4 in M231 shNC group), and few or no tumors were found in mice injected with SETDB1-deficient cells (0/4 in M231 sh2 group; 1/4 in M231 sh3 group) (Fig. 2E). No tumor nodules were observed in other tissues in shNC and shSETDB1 groups.

SETDB1 acts as an EMT inducer by activating Snail expression. MDA-MB-231 cell morphology changed from long spindle shapes to round shapes following knockdown of SETDB1 expression (Fig. 3A). Thus, SETDB1 may be involved EMT in breast cancer. In SETDB1-overexpressing MCF7 cells, there was no difference between MCF7/SETDB1 and MCF7/NC cells after puromycin treatment for 2 weeks. However, most MCF7/SETDB1 cells changed from epithelial to mesenchymal phenotypes on the 26th day (Fig. 3A). Immunoblotting data revealed that E-cadherin and $\beta$-catenin protein was significantly decreased in MCF7/SEDB1 cells on the 26th day after infection and selection, with no difference was observed on the 14th day. By contrast, expression of vimentin was significantly increased in MCF7/SETDB1 cells on the 26th and 14th days (Fig. 3B). Thus, SETDB1 overexpression induced EMT in breast cancer cells. SETDB1 RNA and protein levels decreased after EMT formation on the 26th day compared with the 14th day in the MCF/SETDB1 cells (Fig. 3C). To further address the mechanism of how SETDB1 induces EMT in breast cancer, RT-qPCR to detect the RNA level of EMT markers, including TGF1, ZEB1, Snail, Slug and ZO-1. Slug expression was significantly increased following SETDB1 overexpression in MCF7 cells (Fig. 3D). Western blot analysis revealed no significant difference in Slug protein expression on the 26 and 14th days, whereas Snail protein was significantly increased in MCF7/SETDB1 cells on the 26th day (Fig. 3E). 
A
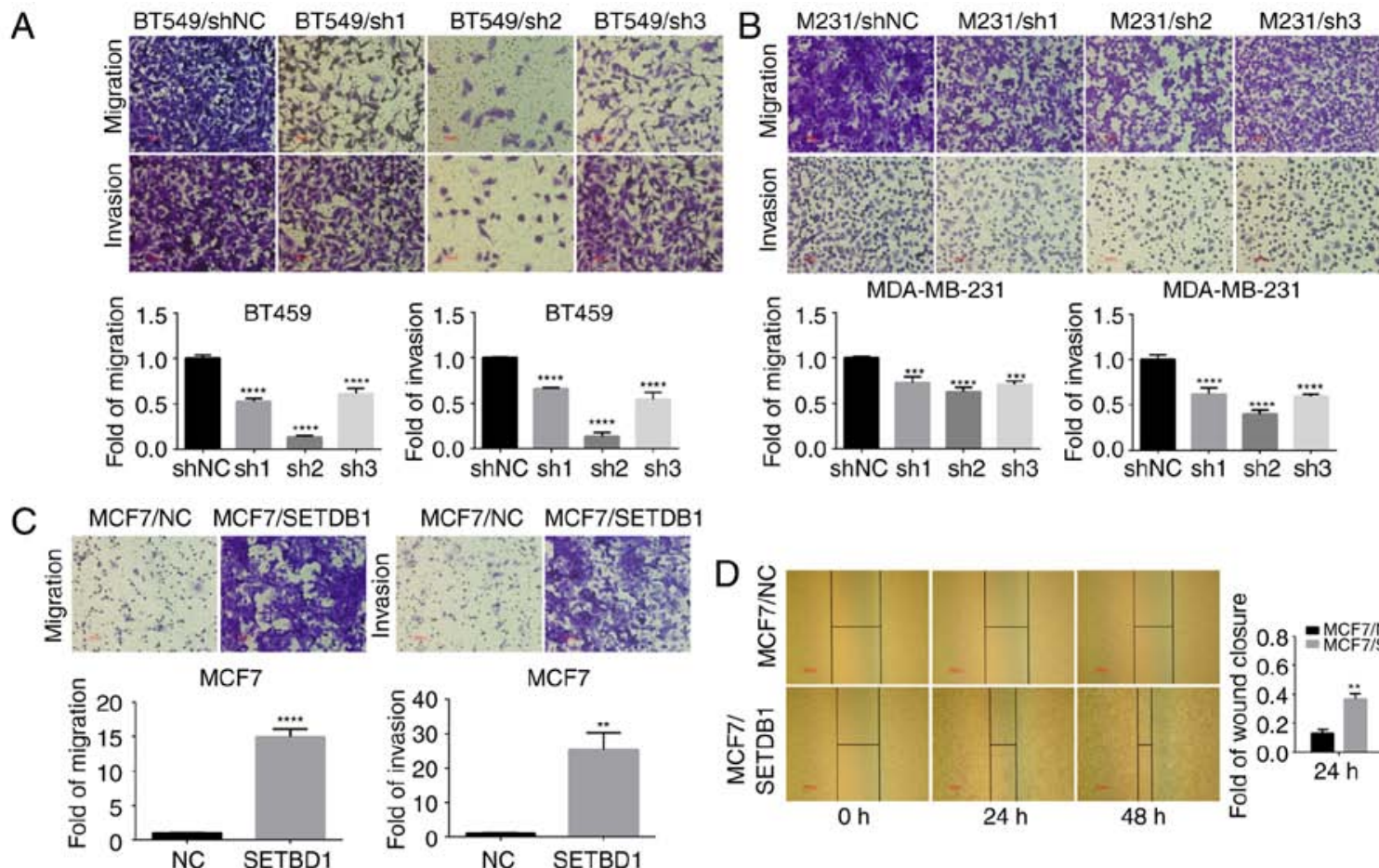

\section{D}

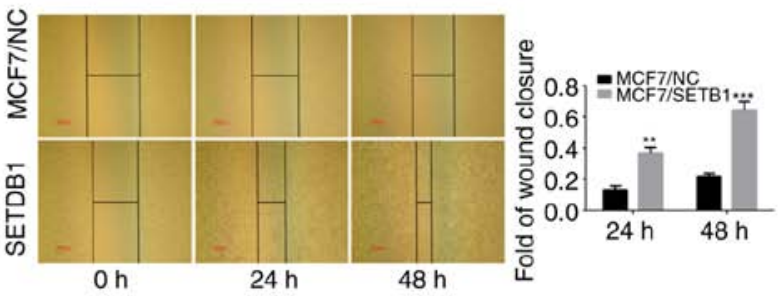

E
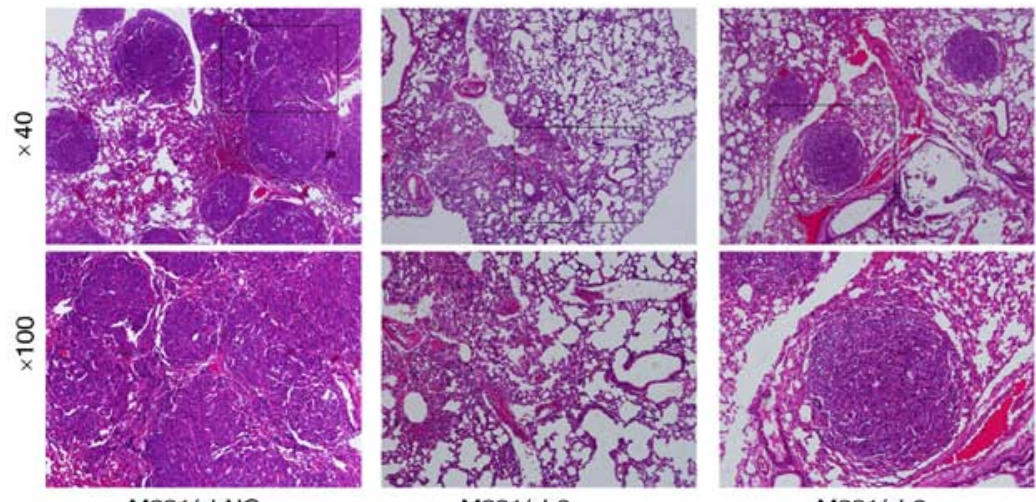

Figure 2. SETDB1 downregulation inhibited breast cancer cell migration and metastasis. (A) Transwell assay data. Knockdown of SETDB1 decreased migration and invasion in BT549 cells after 2 weeks of puromycin selection. Magnification, x200. (B) Knockdown of SETDB1 decreased migration and invasion in MDA-MB-231 cells. (C) Overexpression of SETDB1 significantly increased migration and invasion in MCF7 cells. Magnification, x200. (D) Wound closure was faster in MCF7/SETDB1 cells at 24 and $48 \mathrm{~h}$, after 2 weeks of puromycin selection. Magnification, x40. (E) Hematoxylin and eosin staining of lungs revealed that SETDB1 downregulation significantly suppressed MDA-MB-231 cells ability of metastasis. Data are presented as the means \pm standard deviation of three independent experiments. ${ }^{* *} \mathrm{P}<0.01,{ }^{* * *} \mathrm{P}<0.001,{ }^{* * * *} \mathrm{P}<0.0001$ vs. shNC or NC. sh, short hairpin RNA; NC, negative control; SETDB1, SET domain bifurcated 1 .

Subsequently, CHIP was performed to investigate whether SETDB1 regulated the Snail gene by directly binding to its promoter region. The nucleotide segments from $0-2,000 \mathrm{bp}$ downstream from the initiation start site (ATG) were divided into 13 regions. An enrichment of fragments that hybridized with the CHIP-grade SETDB1 antibody was observed in the promoter regions of P2, P3, P4, P5, P6 and P7 (Fig. 3F). Thus, SETDB1 may be involved in EMT by upregulating the expression of Snail transcription factor.

Knockout of Snail with Crispr-Cas9 partly reversed SETDB1-induced EMT. Following Crispr-Cas9 knockout of Snail expression in SETDB1-overexpressing MCF7 cells, MCF7/SETDB1 cell morphology changed to an epithelial phenotype following Snail knockout, whereas this did not occur in the Crispr-NC group (Fig. 4A). Thus, depletion of Snail may reverse EMT in SETDB1-overexpressing MCF7 cells. Cell migration and invasiveness was significantly decreased in MCF7/SETDB1 + Crispr-Snail cells compared with MCF7/SETDB1 and MCF7/SETDB1 + Crispr-NC cells (Fig. 4B). Immunoblotting results revealed that E-cadherin and $\beta$-catenin protein were significantly increased following knockout of Snail expression, and vimentin was decreased in MCF7/SETDB1 + Crispr-Snail cells (Fig. 4C). These results highlight a critical role of Snail in SETDB1-induced EMT of breast cancer cells. However, compared to MCF7/NC group cells, the expression of E-cadherin and $\beta$-catenin was still significantly decreased and vimentin was increased in MCF7/SETDB1 + Crispr-Snail cells (Fig. 4C). 
A

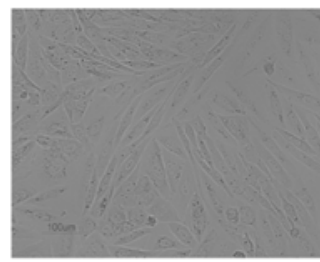

M231/shNC

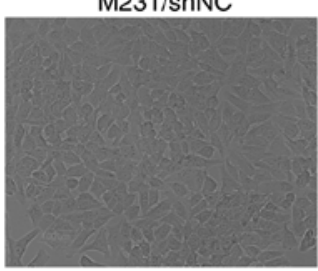

MCF7/NC

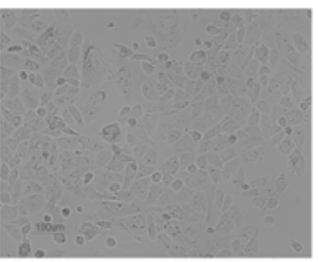

M231/shSETDB1

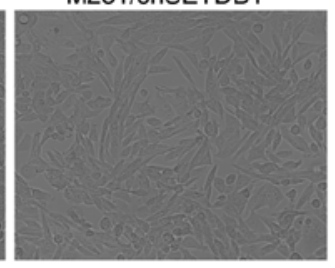

MCF7/SETDB1

\section{C}

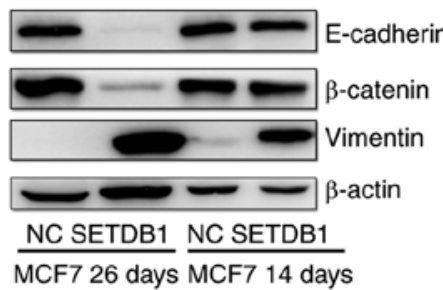

E

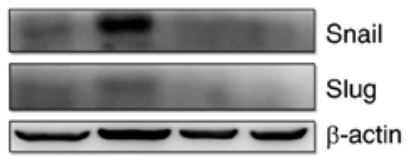

NC SETDB1 NC SETDB1 MCF7 26 days MCF7 14 days
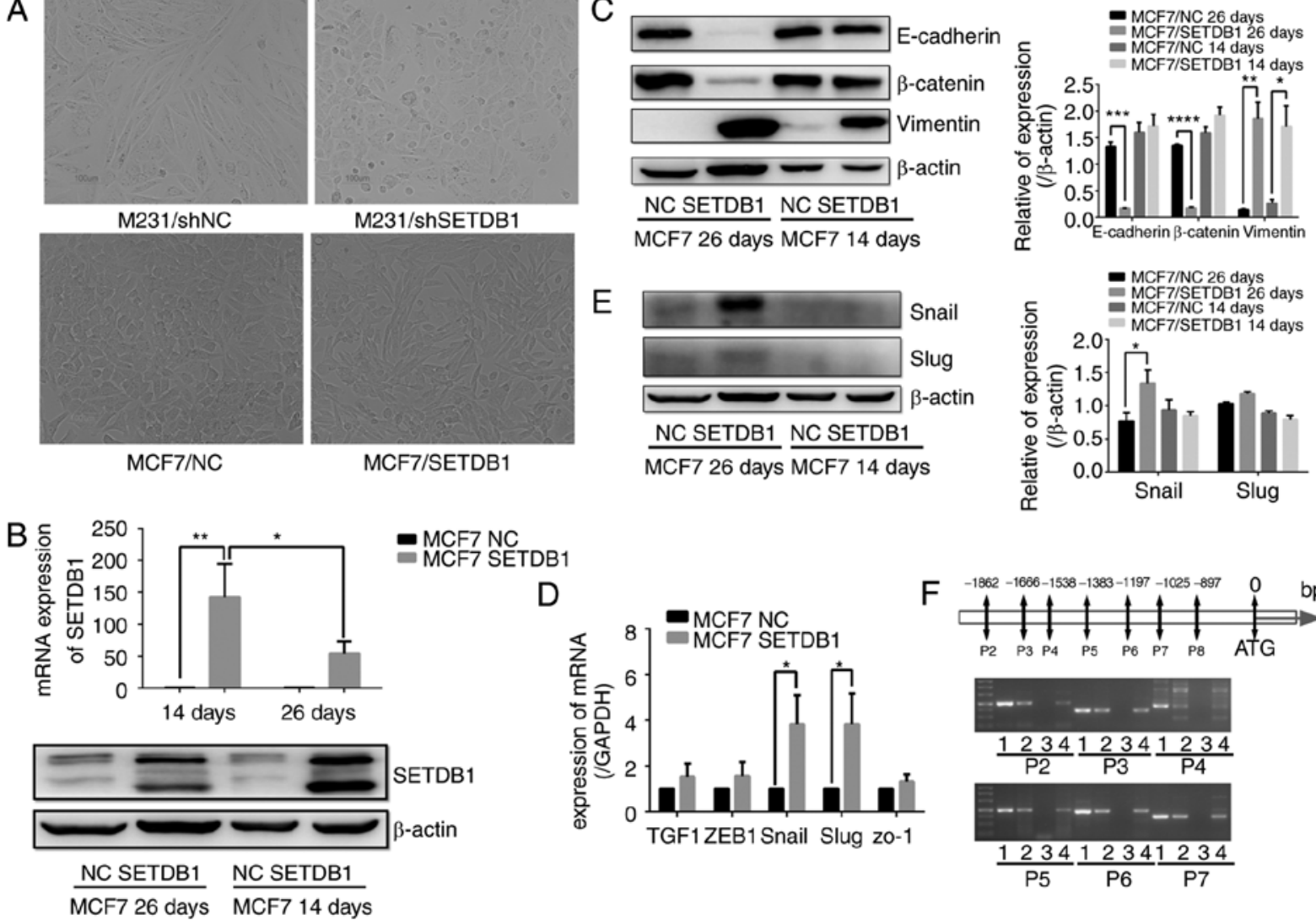

F
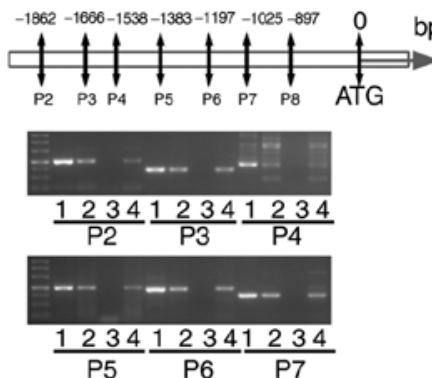

Figure 3. SETDB1 induces EMT by increasing Snail expression. (A) Alterations in the morphology of the MDA-MB-231 at day 14 and MCF7 cells day 26 after puromycin selection were observed following knockdown and overexpression of SETDB1; magnification, x200. (B) Immunoblot of decreased E-cadherin and $\beta$-catenin protein in SETDB1-overexpressing MCF7 cells after 26 days of puromycin selection, but no difference at 14 days. Vimentin expression was significantly increased in MCF7/SETDB1 cells at 26 and 14 days after puromycin selection. (C) SETDB1 RNA (upper) and protein levels (lower) decreased at 26 days compared to 14 days. (D) Reverse transcription-quantitative polymerase chain reaction validation expression of genes associated with EMT including TCF1, ZEB1, Snail, Slug and ZO-1 after SETDB1 upregulation in MCF7 cells; Slug expression was increased in MCF7/SETDB1 cells. (E) Snail protein was significantly increased in MCF7/SETDB1 cells at day 26 but not day 14. Slug protein showed no difference on either day. Data are presented as the mean \pm standard deviation and represent three independent experiments. (F) An enrichment of fragments hybridized with the CHIP-grade SETDB1 antibody was observed in the Snail promoter regions of P2, P3, P4, P5, P6 and P7 in groups 1 (input group), 2 (H3 group), 3 (IgG group) and 4 (SETDB1 group). "P<0.05, "* P<0.01, ${ }^{* * * *} \mathrm{P}<0.001,{ }^{* * * *} \mathrm{P}<0.0001$ vs. NC. EMT, epithelial-mesenchymal transition; sh, short hairpin RNA; NC, negative control; SETDB1, SET domain bifurcated 1; TGF1, transforming growth factor 1; ZEB1, zinc finger E-box binding homeobox 1; ZO-1, tight junction protein 1.

\section{Discussion}

The SETDB1 gene is located at human chromosome 1q21, and many recurrent translocations occur in this region (5). The gene encodes SETDB1 protein, which contains an unusual bifurcated SET domain, suggesting that SET domains may have two distinct functional domains (5). SETDB1, which acts as a histone lysine methyltransferase (HMT), is involved in transcriptional repression and embryonic development (8-15). SETDB1 was demonstrated to be an important oncogene in a zebrafish melanoma model (16). Other studies suggest that SETDB1 overexpression increases cell growth and migration in gliomagenesis and prostate cancer $(17,18)$. Notably, studies have indicated that SETDB1 was amplified in lung cancer, and enhanced cell growth via the WNT signaling pathway and downregulated p53 expression $(19,26)$. However, SETDB1 has been reported to interact with the mothers against decapentaplegic homolog 2/3 (Smad2/3) repressor complex on the Annexin A2 promoter to suppress lung cancer metastasis (27). Thus, further investigation is required to uncover the function and mechanism underlying the role of SETDB1 in cancer.
According to a meta-analysis, five HMTs, including SETDB1, were amplified in $>10 \%$ of breast cancer samples, particularly for triple-negative breast cancer (28). In the present study, SETDB1 expression was detected in breast cancer cell lines, and demonstrated that SETDB1 protein and mRNA were overexpressed in the MCF7 and T47D cells compared to the immortalized mammalian epithelial cell line MCF10A (data not shown). However, compared to its upregulation in MCF7 and T47D, which have low invasion capability, the high level of invasiveness of the breast cancer cells showed lower levels of SETDB1 expression (data not shown), which suggests that SETDB1 may play different roles in the development of breast cancer via multiple mechanisms. Downregulation of SETDB1 in the BT549 and MDA-MB-231 cells reduced cell proliferation, whereas SETDB1 upregulation in MCF7 and T47D cells enhanced proliferation. Recently, Regina et al (29) reported that SETDB1 is overexpressed in breast cancer cell lines and enhanced tumor cell growth, which is consistent with the findings of the current study. Additionally, depletion of SETDB1 suppressed cell migration and invasion in vitro and reduced lung metastasis in vivo. By contrast, SETDB1 overexpression 
A

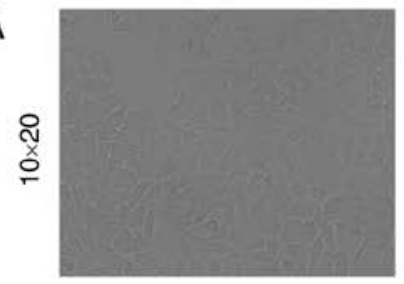

MCF7/NC

B

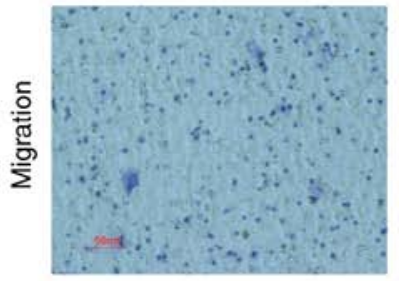

MCF7/NC

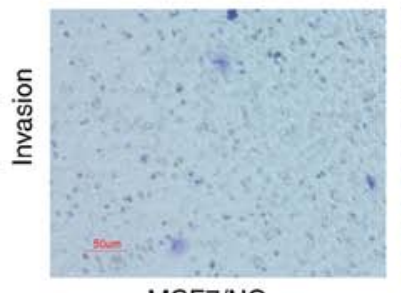

MCF7/NC

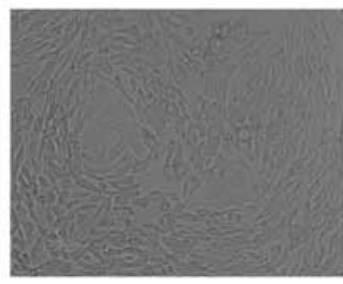

MCF7/SETDB1

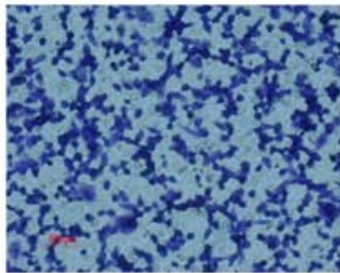

MCF7/SETDB1

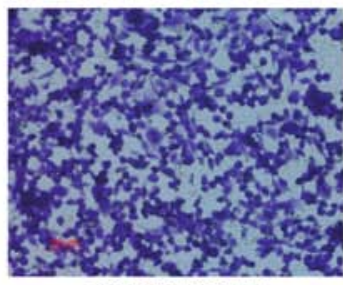

MCF7/SETDB1

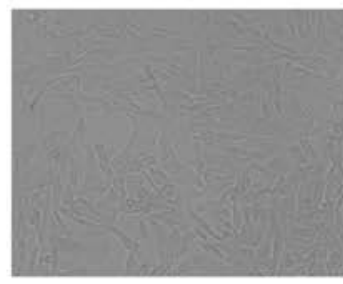

MCF7/SETDB1+Crispr-NC

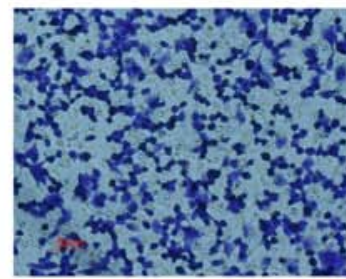

MCF7/SETDB1+Crispr-NC

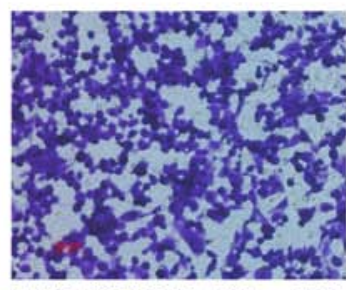

MCF7/SETDB1+Crispr-NC

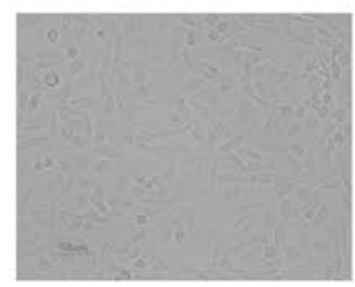

MCF7/SETDB1+Crispr-Snail

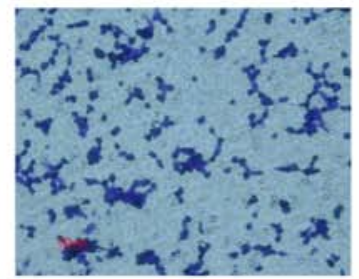

MCF7/SETDB1+Crispr-Snail

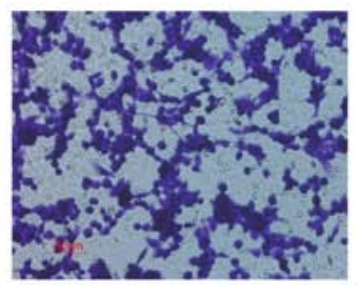

MCF7/SETDB1+Crispr-Snai
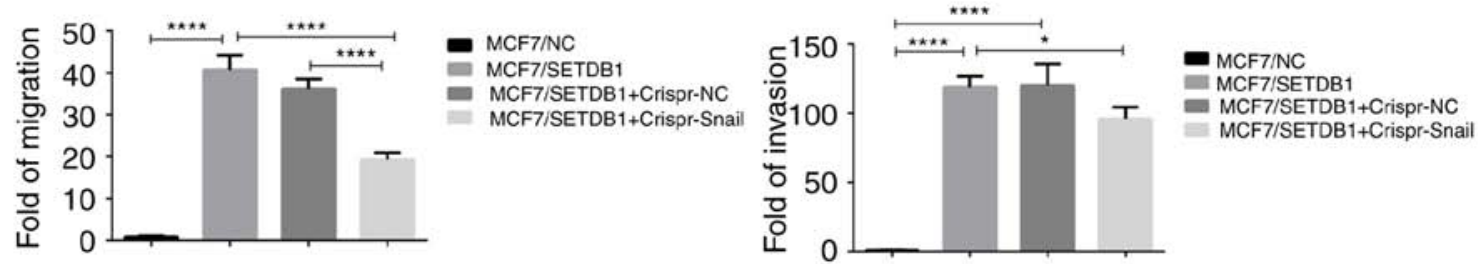

C

MCF7
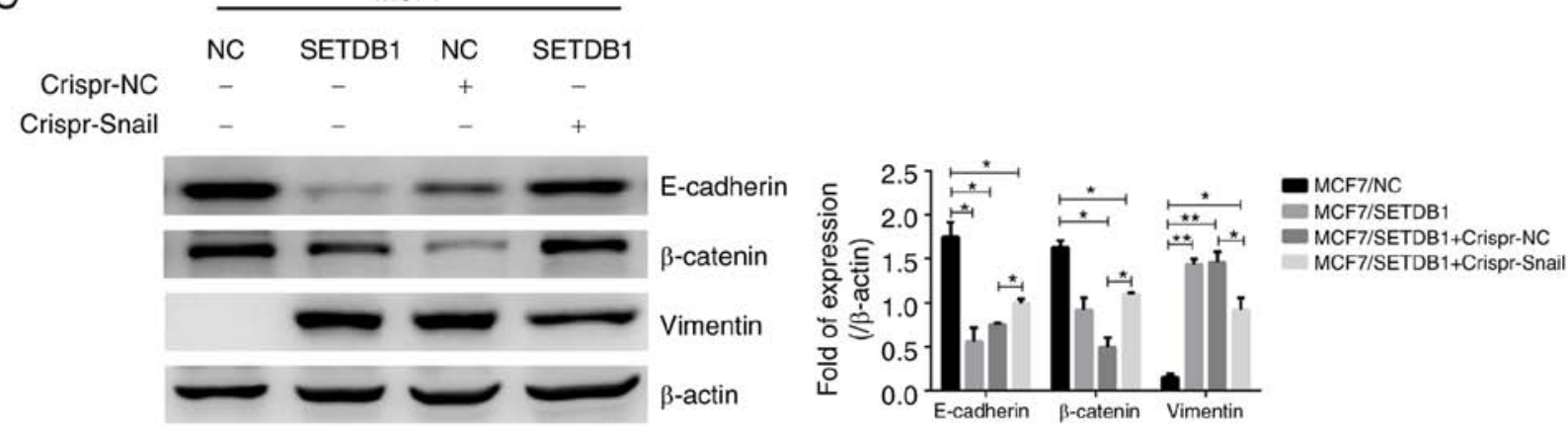

Figure 4. Knockout of Snail via Crispr-Cas9 partially reversed SETDB1-induced EMT. (A) Morphology of MCF7/SETDB1 cells changed from mesenchymal to epithelial phenotypes following Snail knockdown; x200. (B) Compared with the MCF7/NC group, the migration and invasion significantly increased in the MCF7/SETDB1 group $\left({ }^{* * * * *} \mathrm{P}<0.0001\right)$. However, depletion of Snail by Crispr-Cas9 decreased migration $\left({ }^{* * * *} \mathrm{P}<0.0001\right)$ and invasion $\left({ }^{*} \mathrm{P}<0.05\right)$ of MCF7/SETDB1 cells. The red scale bar shows $50 \mu \mathrm{m}$. Magnification, x200. (C) Immunoblot of EMT markers E-cadherin, $\beta$-catenin and vimentin protein in SETDB1-overexpressing and Snail-Crispr knockdown MCF7 cells. Data presented as the mean \pm standard deviation and represent three independent experiments. ${ }^{*} \mathrm{P}<0.05,{ }^{* *} \mathrm{P}<0.01,{ }^{* * * * *} \mathrm{P}<0.0001$. EMT, epithelial-mesenchymal transition; NC, negative control; SETDB1, SET domain bifurcated 1; Crispr, clustered regularly interspaced short palindromic repeats.

enhanced cell migration and invasiveness. In a nude mice model, SETDB1 downregulation significantly suppressed the distant metastasis ability of breast cancer cell in lungs. Thus, SETDB1 has an oncogenic role in breast cancer, but how it causes metastasis is unclear. A previous study reported that microRNA-7 directly inhibits SETDB1 expression, which leads to a reduction in STAT3 expression and partially reverses EMT in breast cancer stem cells (30). Additionally, SETDB1 is a novel interactor of $\Delta \mathrm{Np} 63$ and contributes to stable p63 protein in breast cancer (29).

SETDB1 was identified as an EMT inducer in breast cancer cells. EMT is a process by which cells acquire molecular alterations that facilitate dysfunctional cell-cell adhesive interactions and junctions, and a more spindle-shaped morphology (31). SETDB1 knockdown lead to MDA-MB-231 cell morphological changes from long spindle to round shapes. Furthermore, 
the morphology of SETDB1-overexpression MCF7 cells changed from epithelial to a mesenchymal phenotype following lentiviral-vector infection for 26 days. Additionally, Transwell assay and wound healing assay were performed to detect cell migration and invasion, and protein and RNA were extracted to detect the expression of EMT-associated markers. Immunoblotting revealed that epithelial markers, E-cadherin and $\beta$-catenin, were significantly decreased in MCF7/SEDB1 cells, and mesenchymal marker, vimentin, was significantly increased. Notably, vimentin expression was significantly increased following EMT (day 26), and also at the beginning of the transition (day 14). It is speculated that SETDB1 increased the abilities of migration and invasion mainly via increasing vimentin expression in MCF7 cells. Taken together, these findings demonstrate that SETDB1 is involved in the EMT process in breast cancer. However, changes in the SETDB1 expression in MCF7 cells were not detected until the 26th day after infection with vectors. This finding suggests that the effects of SETDB1 overexpression on inducement of EMT in breast cancer involve a long process. EMT is induced by various signaling pathway such as TGF- $\beta$ (32), bone morphogenetic protein (33), Hedgehog, WNT- $\beta$-catenin and Notch signaling (34). These signaling pathways induce multiple transcription factors during EMT. For instance, ZEB1, ZEB2, and Snail, are induced by TGF- $\beta$-signaling and are critical for TGF- $\beta$-induced EMT (35). Expression of Snail was increased following SETDB1 overexpression in MCF7 cells in the current study. Snail family proteins are zinc finger transcription regulators and bind to the E-box motif via conserved $\mathrm{C} 2 \mathrm{H} 2$ domains (36). Additionally, Snail is a strong transcriptional repressor of the E-cadherin gene and induces epithelial-mesenchymal conversion in epithelial cells (37). A previous study reported that zinc finger protein 274 , which contains five $\mathrm{C} 2 \mathrm{H} 2$ domain binding sites, co-localizes with SETDB1, KAP1 and H3K9me3 at the 3'-ends of zinc finger genes (38). To investigate the interaction between SETDB1 and Snail, CHIP was performed and demonstrated that SETDB1 binds to the promoter of Snail. Furthermore, knocking down Snail expression with Crispr-Cas9 partially reversed SETDB1-induced EMT. Unexpectedly, the expression of E-cadherin and $\beta$-catenin were different between the MCF7/SETDB1 and MCF7/SETDB1-Crispr-NC groups. It was speculated that Cripsr vector infection had a marginal effect on the state of cells, which may contributes to the differences. However, compared with the MCF7/NC group, E-cadherin and $\beta$-catenin protein levels were still significantly decreased. As SETDB1 was stably expressed in MCF7 cells, there were no off target effects caused by the Crispr control vector. Notably, the expression of EMT-associated markers remained significantly different in MCF7/SETDB1 + Crispr-Snail cells compared with the MCF7/NC group cells. These findings indicated that SETDB1 induces EMT via multiple pathways, not only by regulating Snail. Recently, Du et al (39) reported that SETDB1 is downregulated in a TGF- $\beta$-induced EMT model and its silencing enhances EMT by repressing Snail. Notably, SETDB1 was detected expression in the MCF7/SETDB1 cells on the 26th and 14th days after infection with overexpression vectors, SETDB1 expression was also decreased following EMT (day 26 compared with day 14). Thus, when the phenotype of epithelial cells changes to mesenchymal, SETDB1 is potentially negatively regulated by another mechanism that leads to reduced SETDB1 expression, and differs from that observed during early and aggressive breast cancer. Alternatively, SETDB1 may have different roles in various stages of breast cancer, which may be associated its unusual bifurcated SET domain. In summary, SETDB1 is required for breast cancer cell proliferation and invasion. Additional investigations on the functional and pathological roles of SETDB1 in breast cancer are required.

\section{Acknowledgements}

Not applicable.

\section{Funding}

The present study was fund by The National Natural Science Foundation of China (grant nos. 81772796 and 81470857) and PhD Research Foundation Affiliated Hospital of Jining Medical University (grant no. 2017-BS-022).

\section{Availability of data and materials}

The dataset generated or analysed during the present study are available from the corresponding author on reasonable request.

\section{Authors' contributions}

WY conceived and designed the study, analysed and interpreted the data and drafted the manuscript. YS and $\mathrm{CH}$ performed cell culture and lentiviral infection, and proliferation, migration, invasion and wound healing assay. LC performed western blot analyses and RT-PCR. DZ performed CHIP assay and standard PCR. KR contributed to Crispr-Cas9 construction and western blot analysis. ZZ contributed to animal experiment and H\&E staining. RZ and $X L$ reviewed the manuscript, supervised the study and were also involved in the conception of the study. All authors read and approved the manuscript and agree to be accountable for all aspects of the research in ensuring that the accuracy or integrity of any part of the work are appropriately investigated and resolved.

\section{Ethics approval and consent to participate}

Animal experiments were approved by the Ethics Committee of Fudan University (Shanghai, China).

\section{Patient consent for publication}

Not applicable.

\section{Competing interests}

The authors declare that they have no competing interests.

\section{References}

1. Benson JR and Jatoi I: The global breast cancer burden. Future Oncol 8: 697-702, 2012. 
2. Gonzalez-Angulo AM, Morales-Vasquez F and Hortobagyi GN: Overview of resistance to systemic therapy in patients with breast cancer. Adv Exp Med Biol 608: 1-22, 2007.

3. Elsheikh SE, Green AR, Rakha EA, Powe DG, Ahmed RA, Collins HM, Soria D, Garibaldi JM, Paish CE, Ammar AA, et al Global histone modifications in breast cancer correlate with tumor phenotypes, prognostic factors, and patient outcome Cancer Res 69: 3802-3809, 2009.

4. Albert $\mathrm{M}$ and Helin K: Histone methyltransferases in cancer. Semin Cell Dev Biol 21: 209-220, 2010.

5. Harte PJ, Wu W, Carrasquillo MM and Matera AG: Assignment of a novel bifurcated SET domain gene, SETDB1, to human chromosome band 1q21 by in situ hybridization and radiation hybrids. Cytogenet Cell Genet 84: 83-86, 1999.

6. Schultz DC, Ayyanathan K, Negorev D, Maul GG and Rauscher FJ III: SETDB1: A novel KAP-1-associated histone $\mathrm{H} 3$, lysine 9-specific methyltransferase that contributes to HP1-mediated silencing of euchromatic genes by KRAB zinc-finger proteins. Genes Dev 16: 919-932, 2002.

7. Verschure PJ, van der Kraan I, de Leeuw W, van der Vlag J, Carpenter AE, Belmont AS and van Driel R: In vivo HP1 targeting causes large-scale chromatin condensation and enhanced histone lysine methylation. Mol Cell Biol 25: 4552-4564, 2005.

8. Wang H, An W, Cao R, Xia L, Erdjument-Bromage H, Chatton B, Tempst P, Roeder RG and Zhang Y: mAM facilitates conversion by ESET of dimethyl to trimethyl lysine 9 of histone $\mathrm{H} 3$ to cause transcriptional repression. Mol Cell 12: 475-487, 2003.

9. Sarraf SA and Stancheva I: Methyl-CpG binding protein MBD1 couples histone $\mathrm{H} 3$ methylation at lysine 9 by SETDB1 to DNA replication and chromatin assembly. Mol Cell 15: 595-605, 2004

10. Ichimura T, Watanabe S, Sakamoto Y, Aoto T, Fujita N and Nakao M: Transcriptional repression and heterochromatin formation by MBD1 and MCAF/AM family proteins. J Biol Chem 280: 13928-13935, 2005

11. Bilodeau S, Kagey MH, Frampton GM, Rahl PB and Young RA SetDB1 contributes to repression of genes encoding developmental regulators and maintenance of ES cell state. Genes Dev 23: 2484-2489, 2009.

12. Liu S, Brind'Amour J, Karimi MM, Shirane K, Bogutz A, Lefebvre L, Sasaki H, Shinkai Y and Lorincz MC: SetdbI is required for germline development and silencing of H3K9me3-marked endogenous retroviruses in primordial germ cells. Genes Dev 29: 108, 2015.

13. Koide S, Oshima M, Takubo K, Yamazaki S, Nitta E, Saraya A Aoyama K, Kato Y, Miyagi S, Nakajima-Takagi Y, et al: Setdb1 maintains hematopoietic stem and progenitor cells by restricting the ectopic activation of nonhematopoietic genes. Blood 128 638-649, 2016.

14. Thompson PJ, Dulberg V, Moon KM, Foster LJ, Chen C, Karimi MM and Lorincz MC: hnRNP K coordinates transcriptional silencing by SETDB1 in embryonic stem cells. PLoS Genet 11: e1004933, 2015.

15. Fei Q, Yang X, Jiang H, Wang Q, Yu Y, Yu Y, Yi W, Zhou S, Chen T, Lu C, et al: SETDB1 modulates PRC2 activity at developmental genes independently of H3K9 trimethylation in mouse ES cells. Genome Res 25: 1325-1335, 2015

16. Ceol CJ, Houvras Y, Jane-Valbuena J, Bilodeau S, Orlando DA, Battisti V, Fritsch L, Lin WM, Hollmann TJ, Ferré F, et al: The histone methyltransferase SETDB1 is recurrently amplified in melanoma and accelerates its onset. Nature 471: 513-517, 2011.

17. Spyropoulou A, Gargalionis A, Dalagiorgou G, Adamopoulos C, Papavassiliou KA, Lea RW, Piperi C and Papavassiliou AG: Role of histone lysine methyltransferases SUV39H1 and SETDB1 in gliomagenesis: Modulation of cell proliferation, migration, and colony formation. Neuromolecular Med 16: 70-82, 2014.

18. Sun Y, Wei M, Ren SC, Chen R, Xu WD, Wang FB, Lu J, Shen J, Yu YW, Hou JG, et al: Histone methyltransferase SETDB1 is required for prostate cancer cell proliferation, migration and invasion. Asian J Androl 16: 319-324, 2014.

19. Rodriguez-Paredes M, Martinez de Paz A, Simó-Riudalbas L, Sayols S, Moutinho C, Moran S, Villanueva A, VázquezCedeira M, Lazo PA, Carneiro F, et al: Gene amplification of the histone methyltransferase SETDB1 contributes to human lung tumorigenesis. Oncogene 33: 2807-2813, 2014.

20. Chen K, Zhang F, Ding J, Liang Y, Zhan Z, Zhan Y, Chen LH and Ding Y: Histone methyltransferase SETDB1 promotes the progression of colorectal cancer by inhibiting the expression of TP53. J Cancer 8: 3318-3330, 2017.
21. Ho Y, Lin YM, Huang YC, Chang J, Yeh KT, Lin LI, Gong Z, Tzeng TY and Lu JW: Significance of histone methyltransferase SETDB1 expression in colon adenocarcinoma. APMIS 125: 985-995, 2017.

22. Noh HJ, Kim KA and Kim KC: p53 Down-regulates SETDB1 gene expression during paclitaxel induced-cell death. Biochem Biophys Res Commun 446: 43-48, 2014

23. Cicchini $\mathrm{C}$, Battistelli $\mathrm{C}$ and Tripodi M: SETDB1 is a new promising target in HCC therapy. Chin Clin Oncol 5: 73, 2016.

24. Karanth AV, Maniswami RR, Prashanth S, Govindaraj H, Padmavathy R, Jegatheesan SK, Mullangi R and Rajagopal S: Emerging role of SETDB1 as a therapeutic target. Expert Opin Ther Targets 21: 319-331, 2017.

25. Yang W, Wang JG, Xu J, Zhou D, Ren K, Hou C, Chen L and Liu X: HCRP1 inhibits TGF- $\beta$ induced epithelial-mesenchymal transition in hepatocellular carcinoma. Int J Oncol: Mar 8, 2017 (Epub ahead of print). doi: 10.3892/ijo.2017.3903.

26. Sun QY, Ding LW, Xiao JF, Chien W, Lim SL, Hattori N, Goodglick L, Chia D, Mah V, Alavi M, et al: SETDB1 accelerates tumourigenesis by regulating the WNT signalling pathway. J Pathol 235: 559-570, 2015.

27. Wu PC, Lu JW, Yang JY, Lin IH, Ou DL, Lin YH, Chou KH, Huang WF, Wang WP, Huang YL, et al: H3K9 histone methyltransferase, KMT1E/SETDB1, cooperates with the SMAD2/3 pathway to suppress lung cancer metastasis. Cancer Res 74: 7333-7343, 2014

28. Liu L, Kimball S, Liu H, Holowatyj A and Yang ZQ: Genetic alterations of histone lysine methyltransferases and their significance in breast cancer. Oncotarget 6: 2466-2482, 2015.

29. Regina C, Compagnone M, Peschiaroli A, Lena A, AnnicchiaricoPetruzzelli M, Piro MC, Melino G and Candi E: Setdb1, a novel interactor of $\Delta \mathrm{Np} 63$, is involved in breast tumorigenesis. Oncotarget 7: 28836-28848, 2016.

30. Zhang H, Cai K, Wang J, Wang X, Cheng K, Shi F, Jiang L, Zhang Y and Dou J: MiR-7, inhibited indirectly by lincRNA HOTAIR, directly inhibits SETDB1 and reverses the EMT of breast cancer stem cells by downregulating the STAT3 pathway. Stem Cells 32: 2858-2868, 2014.

31. Creighton CJ, Chang JC and Rosen JM: Epithelial-mesenchymal transition (EMT) in tumor-initiating cells and its clinical implications in breast cancer. J Mammary Gland Biol Neoplasia 15: 253-260, 2010.

32. Kahata K, Dadras MS and Moustakas A: TGF- $\beta$ family signaling in epithelial differentiation and epithelial-mesenchymal transition. Cold Spring Harb Perspect Biol 10: a022194, 2018

33. McCormack N and O'Dea S: Regulation of epithelial to mesenchymal transition by bone morphogenetic proteins. Cell Signal 25: 2856-2862, 2013.

34. Zardawi SJ, O'Toole SA, Sutherland RL and Musgrove EA: Dysregulation of Hedgehog, Wnt and Notch signalling pathways in breast cancer. Histol Histopathol 24: 385-398, 2009.

35. Miyazono K: Transforming growth factor-beta signaling in epithelial-mesenchymal transition and progression of cancer. Proc Jpn Acad Ser B Phys Biol Sci 85: 314-323, 2009.

36. Kataoka H, Murayama T, Yokode M, Mori S, Sano H, Ozaki H, Yokota Y, Nishikawa S and Kita T: A novel snail-related transcription factor Smuc regulates basic helix-loop-helix transcription factor activities via specific E-box motifs. Nucleic Acids Res 28: 626-633, 2000

37. Cano A, Pérez-Moreno MA, Rodrigo I, Locascio A, Blanco MJ, del Barrio MG, Portillo F and Nieto MA: The transcription factor snail controls epithelial-mesenchymal transitions by repressing E-cadherin expression. Nat Cell Biol 2: 76-83, 2000.

38. Frietze S, O'Geen H, Blahnik KR, Jin VX and Farnham PJ: ZNF274 recruits the histone methyltransferase SETDB1 to the 3' ends of ZNF genes. PLoS One 5: e15082, 2010.

39. Du D, Katsuno Y, Meyer D, Budi EH, Chen SH, Koeppen H, Wang H, Akhurst RJ and Derynck R: Smad3-mediated recruitment of the methyltransferase SETDB1/ESET controls Snail1 expression and epithelial-mesenchymal transition. EMBO Rep 19: 135-155, 2018. 